\title{
On PVPG graphs: a subclass of vertex intersection graphs of paths on a grid
}

\author{
Liliana Alcón, Flavia Bonomo-Braberman, \\ María Pía Mazzoleni, ${ }^{(1)}$ Fabiano de Souza Oliveira ${ }^{(1)}$
}

\begin{abstract}
In this paper, we study the class of PVPG graphs, this is a subclass of VPG graphs such that all the representing paths are between two parallel lines of the grid and have their endpoints on these lines. We prove that PVPG = Co-comparability. Moreover, we present some minimal forbidden induced subgraphs for the class of $B_{1}-\mathrm{PVPG}$ graphs.
\end{abstract}

\section{Introduction}

A $V P G$ representation of a graph $G$ is a collection of paths of the twodimensional grid where the paths represent the vertices of $G$ in such a way that two vertices of $G$ are adjacent in $G$ if and only if the corresponding paths share at least one vertex of the grid. A graph which has a VPG representation is called a VPG graph. The recognition problem is NPcomplete for VPG graphs [1]. For applications of VPG graphs see [6, 2, 5].

A graph $G$ is a Comparability graph if there exists a poset $\mathbf{P}$ with the same vertex set as $G$ such that two vertices are comparable in $\mathbf{P}$ if and

2000 AMS Subject Classification: 05C62, 05C75.

Keywords and Phrases: Vertex intersection graphs, grids, Co-comparability graphs. This research was partially supported by FAPERJ and UBACyT. 
only if they are adjacent in $G$. Or equivalently, if it is possible to orient the edges of $G$ in such a way that the resultant digraph has the transitivity property: if edges $u \rightarrow v$ and $v \rightarrow w$ exist, then so does the edge $u \rightarrow w$. We say that $G$ is a Co-comparability graph when $\bar{G}$ (the complement of $G)$ is a Comparability graph.

In [4, the authors show that the class of Co-comparability graph is a subclass of VPG graphs. In this work we present the PVPG graphs defined by the property of admitting a VPG representation in which all paths are between, and have their endpoints on, two given parallel lines of the grid. We prove that this subclass of VPG is exactly the class of Cocomparability graphs. We study characteristics of PVPG representation that we believe will be fundamental tools for the development of future work. A $B_{k}-P V P G$ representation is a PVPG representation in which each path in the representation has at most $k$ bends. We present some minimal forbidden induced subgraphs for a graph to admit a $\mathrm{B}_{1}$-PVPG representation. Finally we posed some open problems.

Definitions not included in this work due to space limitations, can be found in [3].

\section{PVPG graphs}

First we introduce formally the class of PVPG graphs.

Definition 2.1. A PVPG representation of a graph $G$, is a VPG representation in which all paths run between, and have their end vertices on, two parallel lines of the grid (see examples in each Figure of this work). A PVPG graph is a graph which has a PVPG representation.

Next we prove that PVPG graphs are exactly Co-comparability graphs.

Theorem 2.1. $P V P G=$ Co-comparability.

Proof. Let $G$ be a PVPG graph and let $\langle\mathcal{P}, \mathcal{G}\rangle$ be a PVPG representation of $G$. We have to see that $G$ is a Co-comparability graph. It is equivalent 

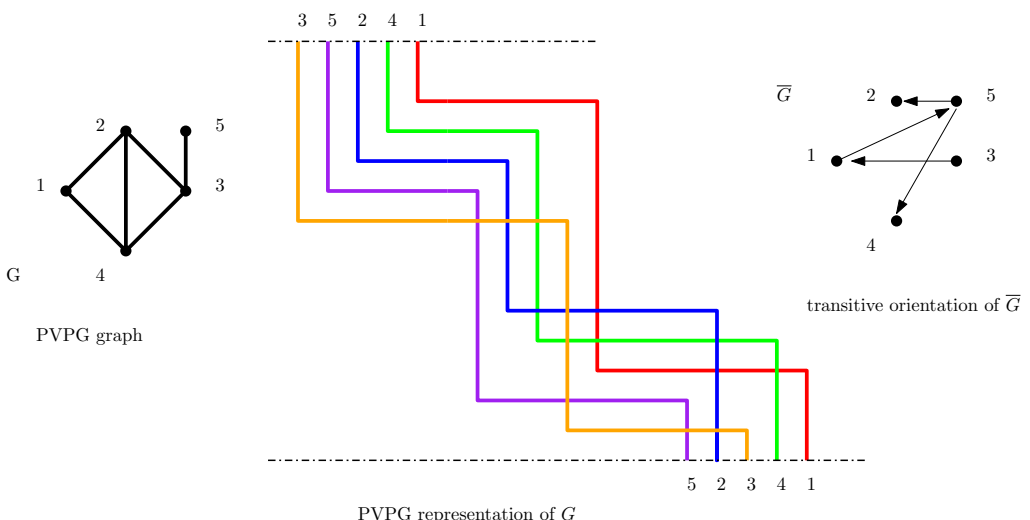

Figure 1: Transitive orientation of $\bar{G}$.

to see that $\bar{G}$ is a Comparability graph, that is, $\bar{G}$ has a transitive orientation of its edges. Let $u, v \in V(\bar{G})$. If $u v \in E(\bar{G})$, then $u v \notin E(G)$. Thus we have that, in $\langle\mathcal{P}, \mathcal{G}\rangle, P_{u}$ is totally to the left of $P_{v}$ (or vice versa). We are going to give a transitive orientation of the edges of $\bar{G}$ in the following way: if $u v \in E(\bar{G})$ such that $P_{u}$ is totally to the left of $P_{v}$, we orient the edge $u v$ as $u \rightarrow v$ (if $P_{u}$ is totally to the right of $P_{v}$ we orient the edge $u v$ as $u \leftarrow v)$. Hence, $\bar{G}$ is a comparability graph and the proof follows. See an example in Figure 1 .

Now, let $G$ be a Co-comparability graph, thus $\bar{G}$ is the comparability graph of some poset $\mathbf{P}$, which in turn is the intersection of some linear orders. Using them, its is possible to build a PVPG representation of $G$ as shown in the example in Figure 2. For more details on this construction, refer to 4].

Corollary 2.1. $P V P G \subseteq C_{5}$-free $\cap A T$-free.

Proof. It is well known that Co-comparability $\subseteq C_{5}$-free $\cap A T$-free. Hence, using Theorem 2.1, the proof follows.

A $\mathrm{B}_{k}$-VPG representation is a VPG representation using paths with at most $k$ bends. The bend number of a $V P G$ graph $G$, denoted by $b(G)$, is 


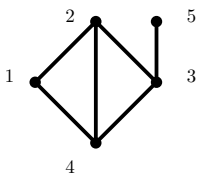

$G$ co-comparability

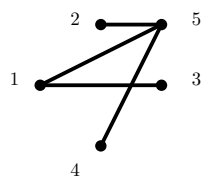

$\bar{G}$ comparability

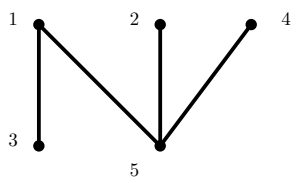

Poset $\mathbf{P}$ whose comparablity graph is $\bar{G}$
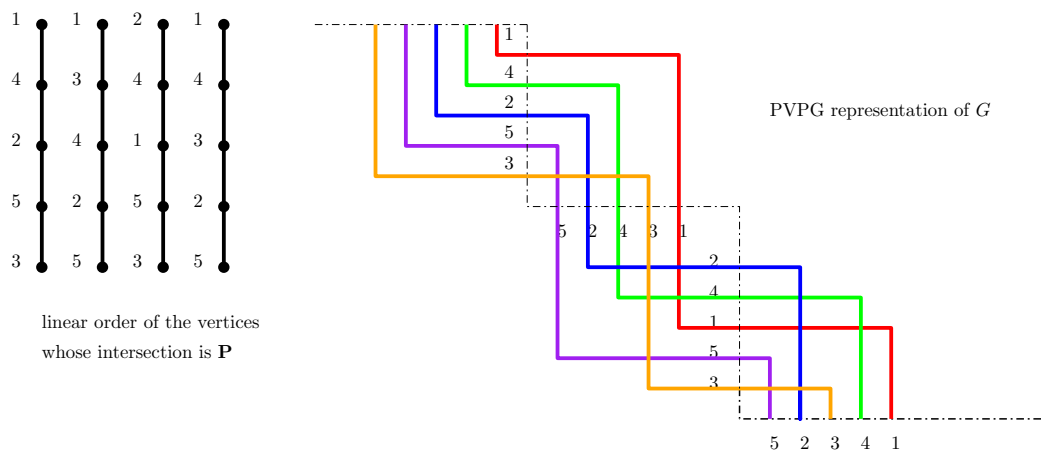

Figure 2: Every co-comparability graph is PVPG.

the minimum integer $k$ for which $G$ has a $\mathrm{B}_{k}$-VPG representation. In an analogous way, we define the parallel bend number.

Definition 2.2. A $B_{k}-P V P G$ representation is a $P V P G$ representation using paths with at most $k$ bends. The parallel bend number of a PVPG graph $G$, denoted by $b_{p}(G)$, is the minimum integer $k$ for which $G$ has a $B_{k}-P V P G$ representation. We call $B_{k}-P V P G$ to the class of PVPG graphs that have parallel bending number at most $k$.

Remark 2.1. $\quad$ 1. $b(G) \leq b_{p}(G)$ (any PVPG representation is a VPG representation).

2. In general $b(G) \neq b_{p}(G)$ (for example $b\left(K_{3,3}\right)=1$ but $b_{p}\left(K_{3,3}\right)=2$ ).

3. $b_{p}(G)$ can be arbitrarily large ( Co-comparability graphs with arbitrarily large bend number are shown in [4]).

Let $\left(P_{v}\right)_{v \in V}$ be a $B_{1}$-PVPG representation of $G$. We will write $P_{v}<<$ $P_{w}$ meaning that the vertical part of $P_{v}$ is to the left of the vertical part 
of $P_{w}$. We say that $v$ is a left (right) extreme vertex of $G$ if $P_{v}<<P_{w}$ $\left(P_{w}<<P_{v}\right)$ for any other vertex $w$ of $G$. Extreme will mean left or right extreme. We say that $w$ is between $v$ and $u$ when either $P_{v}<<P_{w}<<P_{u}$ or $P_{u}<<P_{w}<<P_{v}$.

The following four lemmas will be used to find four minimal forbidden induced subgraphs for the class $B_{1}$-PVPG. Some proof are omitted due to space limitations.

Lemma 2.1. Let $G$ be the complete bipartite graph $K_{1,4}$ with partition $\{x\} \cup\{1,2,3,4\}$ of its vertex set. In any $B_{1}-P V P G$ representation of $G$, if $P_{1}<<P_{2}<<P_{3}<<P_{4}$ then $x$ is not between 2 and 3 .

Lemma 2.2. If $x$ is adjacent to $y$ and $P_{x}<<P_{v_{1}}<<\ldots<<P_{v_{k}}<<P_{y}$, then there exists $r, 0 \leq r \leq k$, such that $x$ is adjacent to the first $r$ vertices of the sequence $v_{1}, v_{2}, \ldots v_{k}$ and $y$ is adjacent to the last $k-r$ vertices.

Corollary 2.2. If $x$ is adjacent to $y$ and $z$ is neither adjacent to $x$ nor to $y$, then $z$ is not between $x$ and $y$.

Lemma 2.3. Let $G$ be the complete bipartite graph $K_{2,3}$ with partition $\{x\} \cup\{1,2,3,4\}$ of its vertex set. In any $B_{1}-P V P G$ representation of $G$ one of the following conditions holds: $(i) x$ or $y$ is an extreme vertex of $G$ and at least two of the vertices $1,2,3$ is between $x$ and $y ;(i i) P_{1}<<$ $P_{x}<<P_{3}<<P_{y}<<P_{2}$.

Proof. Suppose neither $x$ nor $y$ is extreme, then w.l.o.g. we can assume $P_{1}<<P_{x}<<P_{y}<<P_{2}$. If $P_{3}<<P_{1}$, by Lemma 2.2, using that 3 is adjacent to $y$ and that $y$ is non-adjacent to $x$, we have the contradiction 3 adjacent to 1 . Hence, either $x$ and y are not extreme and $P_{x}<<P_{3}<<$ $P_{y}$, or $x$ (or $y$ ) is an extreme vertex of $G$. If $x$ or $y$ is extreme, then w.l.o.g. we can assume that $x$ is an extreme vertex of $G$. Suppose that only one of the vertices $1,2,3$ is between $x$ and $y$, w.l.o.g. we can assume that $P_{x}<<P_{1}<<P_{y}<<P_{2}<<P_{3}$. But, using that $x$ is adjacent to $3, x$ is non-adjacent to $y$ and 3 is non-adjacent to 2 , this contradicts Lemma 2.2 Hence, at least two of the vertices $1,2,3$ is between $x$ and $y$. The cases 


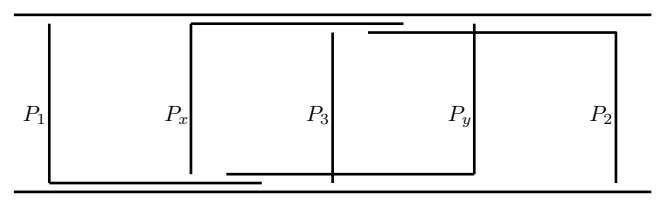

Figure 3: A $B_{1}$-PVPG representation of $K_{2,3}$.

$P_{1}<<P_{3}<<P_{x}, P_{y}<<P_{3}<<P_{2}$ and $P_{2}<<P_{3}$ follows similarly. If $P_{x}<<P_{3}<<P_{y}$, then the model $P_{1}<<P_{x}<<P_{3}<<P_{y}<<P_{2}$ given in Figure 3 is a $B_{1}$-PVPG representation of $G$.

Lemma 2.4. Let $G$ be a chordless path $P_{5}$ with vertices $[x, u, y, v, z]$. In any $B_{1}-P V P G$ representation of $G$ the vertex $y$ is between $x$ and $z$.

In what follows we present four minimal forbidden induced subgraphs for the class of $B_{1}-\mathrm{PVPG}$ graphs.

Theorem 2.2. The complete bipartite graph $K_{3,3}$ is not $B_{1}-P V P G$.

Proof. Let $\{a, b, c\} \cup\{1,2,3\}$ be the partition of $K_{3,3}$. Assume there exists a $B_{1}$-PVPG representation of this graph.

Case (1): By Lemma 2.3, w.l.o.g, we can assume $P_{a}<<P_{1}<<P_{2}<<$ $P_{3}$ and $P_{1}<<P_{b}$, using the $K_{2,3}$ induced by $\{a, b, 1,2,3\}$. Now, applying the result of Lemma 2.3 to the $K_{2,3}$ with partition induced by $\{a, c, 1,2,3\}$ we have that $P_{1}<<P_{c}$. Now, again by Lemma 2.3 , using the $K_{2,3}$ with partition $\{b, c, 1,2,3\}$ we have, w.l.o.g, $P_{a}<<P_{1}<<P_{2}<<P_{3}<<P_{c}$ and $P_{1}<<P_{b}<<P_{3}$. If $P_{1}<<P_{b}<<P_{2}$, we get a contradiction to the result in Lemma 2.2, using $a$ adjacent to 3 . If $P_{2}<<P_{b}<<P_{3}$, we get a contradiction to the result in Lemma 2.2, using 1 adjacent to $c$.

Case (2): $P_{1}<<P_{a}<<P_{3}<<P_{b}<<P_{2}$. By Lemma 2.1, $P_{a}<<$ $P_{c}<<P_{3}$. This contradicts Lemma 2.2, because $a$ is adjacent to 2 but $a$ is non-adjacent to $c$ and 2 is non-adjacent to 3 .

For a vertex $v$, we let $N(v)$ denote the set of vertices adjacent to $v$, and $N[v]=\mathrm{N}(\mathrm{v}) \cup\{\mathrm{v}\}$. 
Theorem 2.3. Let $G$ be the bipartite graph with partition $\{a, b, c\} \cup$ $\{1,2,3,4\}$; and adjacency $N(a)=\{1,2,3\}, N(b)=\{1,2,3,4\}$ and $N(c)=$ $\{2,3,4\}$. Then, $G$ is not $B_{1}-P V P G$.

Proof. Applying Lemma 2.4 to the path $[a, 1, b, 4, c]$, we have that $P_{b}$ is between $P_{a}$ and $P_{c}$. Say, w.l.o.g, that $P_{a}<<P_{b}<<P_{c}$.

Case (1): By Case $(i)$ of Lemma 2.3, using $a, b$ and 1,2,3, we have that between $a$ and $b$ there are two of the vertices 1,2,3. Again, by Case $(i)$ of Lemma 2.3, using $b, c$ and 2,3,4, we have that between $b$ and $c$ there are two of the vertices $2,3,4$, but this contradicts Lemma 2.1 .

Case (2): By Case (ii) of Lemma 2.3, using $a, b$ and 1,2,3, we have $P_{1}<<P_{a}<<P_{3}<<P_{b}<<P_{2}<<P_{c}$. If the other $K_{2,3}$ with partition $b, c$ and 2,3,4 is in Case $(i)$ of Lemma 2.3, then at least two of the vertices 2,3,4 is between $b$ and $c$ which contradicts Lemma 2.1. If the other $K_{2,3}$ with partition $b, c$ and $2,3,4$ is in the case (ii) of Lemma 2.3 , then we have $P_{3}<<P_{b}<<P_{2}<<P_{c}<<P_{4}$ which contradicts Lemma 2.1.

Theorem 2.4. Let $G$ be the bipartite graph with partition $\{a, b, c\} \cup$ $\{1,2,3,4,5\}$; and adjacency $N(a)=\{1,2,3\}, N(b)=\{1,2,3,4,5\}$ and $N(c)=\{3,4,5\}$. Then, $G$ is not $B_{1}-P V P G$.

Proof. Applying Lemma 2.4 to the path $[a, 1, b, 5, c]$, we have that $P_{b}$ is between $P_{a}$ and $P_{c}$. Say, w.l.o.g, that $P_{a}<<P_{b}<<P_{c}$.

Case (1): By Case $(i)$ of Lemma 2.3, using $a, b$ and $1,2,3$, we have that between $a$ and $b$ there are two of the vertices 1,2,3. Again, by Case $(i)$ of Lemma 2.3, using $b, c$ and $3,4,5$, we have that between $b$ and $c$ there are two of the vertices $3,4,5$, but this contradicts Lemma 2.1 .

Case (2): By Case (ii) of Lemma 2.3, using $a, b$ and 1,2,3, we have $P_{1}<<P_{a}<<P_{3}<<P_{b}<<P_{2}<<P_{c}$. If the other $K_{2,3}$ with partition $b, c$ and $3,4,5$ is in Case $(i)$ of Lemma 2.3 , then at least two of the vertices $3,4,5$ is between $b$ and $c$ which contradicts Lemma 2.1. If the other $K_{2,3}$ with partition $b, c$ and $3,4,5$ is in Case (ii) of Lemma 2.3, then we have $P_{3}<<P_{b}<<P_{5}<<P_{c}<<P_{4}$ which contradicts Lemma 2.1. 
Theorem 2.5. Let $G$ be the bipartite graph with partition $\{a, b, c\} \cup$ $\{1,2,3,4,5,6\}$; and adjacency $N(a)=\{1,2,3\}, N(c)=\{4,5,6\}$ and $N(b)=\{1,2,3,4,5,6\}$. Then, $G$ is not $B_{1}-P V P G$.

Proof. Applying Lemma 2.4 to the path $[a, 3, b, 4, c]$, we have that $P_{b}$ is between $P_{a}$ and $P_{c}$. Say, w.l.o.g, that $P_{a}<<P_{b}<<P_{c}$.

Case (1): By Case $(i)$ of Lemma 2.3, using $a, b$ and $1,2,3$, we have that between $a$ and $b$ there are two of the vertices 1,2,3. Again, by Case $(i)$ of Lemma 2.3, using $b, c$ and 4,5,6, we have that between $b$ and $c$ there are two of the vertices $4,5,6$, but this contradicts Lemma 2.1 .

Case (2): By Case (ii) of Lemma 2.3, using $a, b$ and 1,2,3, we have $P_{1}<<P_{a}<<P_{3}<<P_{b}<<P_{2}<<P_{c}$. If the other $K_{2,3}$ with partition $b, c$ and 4,5,6 is in Case $(i)$ of Lemma 2.3 , then at least two of the vertices $4,5,6$ is between $b$ and $c$ which contradicts Lemma 2.1. If the other $K_{2,3}$ with partition $b, c$ and $4,5,6$ is in Case ( $i i)$ of Lemma 2.3, then we have $P_{4}<<P_{b}<<P_{6}<<P_{c}<<P_{5}$ which contradicts Lemma 2.1.

\section{Open problems}

As immediate future work, we are working on finding the complete family of minimal forbidden induced subgraphs for the class $B_{1}$-PVPG.

The well known class of permutation graphs is contained in the class of co-comparability graphs, and so in the class of PVPG graphs. Is it possible to characterize permutation graphs by imposing some restrictions to PVPG-representations? We let as open problem to characterize permutation $\mathrm{B}_{1}-\mathrm{PVPG}$ graphs by induced forbidden subgraphs. We conjecture that a permutation graph is $B_{1}-\mathrm{PVPG}$ if and only if it contains none of the graphs depicted in Theorems 2.2, 2.3, 2.4 and 2.5 as induced subgraphs.

Several questions posed in [4] about the relation between the dimension of a comparability graph and the bend number of its complement, can be extended to the parallel bend number. For instance, a challenging open problem is characterizing the co-comparability graphs such that $\operatorname{dimension}(\bar{G})=b_{p}(G)$. 


\section{References}

[1] A. Asinowski, E. Cohen, M. C. Golumbic, V. Limouzy, M. Lipshteyn and M. Stern, Vertex Intersection Graphs of Paths on a Grid, Journal of Graph Algorithms and Applications 16(2) (2012), 129-150.

[2] M. Bandy and M. Sarrafzadeh, Stretching a knock-knee layout of multilayer wiring, IEEE Trans. Computing 39 (1990), 148-151.

[3] A. Brandstädt, V. Le and J. Spinrad, Graph classes: a survey, (1999), Society for Industrial and Applied Mathematics.

[4] E. Cohen, M. C. Golumbic, W. T. Trotter and R. Wang, Posets and VPG Graphs, Order 33 (2016), 39- 49.

[5] P. Molitor, A survey on wiring, EIK Journal of Information Processing and Cybernetics 27 (1991), 3-19.

[6] F. Sinden, Topology of thin film circuits, Bell System Tech. J. 45 (1966), 1639-1662. 
Liliana Alcón

La Plata, BA, Argentina.

Universidad Nacional de La

Plata, CONICET, Centro de

Matemática Aplicada de La Plata (CEMaLP).

liliana@mate.unlp.edu.ar
Flavia Bonomo-Braberman

Buenos Aires, Argentina

a Universidad de Buenos Aires,

CONICET, Instituto de Investigación en Ciencias de la Computación (ICC).

fbonomo@dc.uba.ar
María Pía Mazzoleni

La Plata, BA, Argentina.

Universidad Nacional de La Universidade do Estado do Rio Plata, CONICET, Centro de de Janeiro.

Matemática Aplicada de La fabiano.oliveira@ime.uerj.br Plata (CEMaLP).

pia@mate.unlp.edu.ar
Fabiano de Souza Oliveira

Rio de Janeiro, Brasil. 